\title{
Transplante cardíaco humano: experiência inicial
}

Noedir A. G. STOLF*, Edimar BOCCHI*, Pedro C. P. LEMOS*, Fábio Biscegli JATENE*, Pablo M. A. POMERANTZEFF*, Lourdes HIGUSHI*, Jorge KALIL*, Alfredo I. FIORELLI*, José Otavio C. AULER JÚNIOR*, Giovanni BELLOTTI*, Lélio A. SILVA*, Fulvio PILEGGI*, Adib D. JATENE*

STOLF, N. A. G.; BOCCHI, E.; LEMOS, P. C. P.; JATENE, F. B.; POMERANTZEFF, P. M. A.; HIGUSHI, L.; KALIL, J.; FIORELLI, A. I.; AULER JUNIOR, J. O. C.; BELLOTTI, G.; SILVA, L. A.; PILEGGI, F.; JATENE, A. D. - Transplante cardiaco humano: experiência inicial. Rev. Bras. Cir. Cardiovasc. 1(2):34-40, 1986.

RESUMO: No Instituto do Coraçāo, de março de 1985 a fevereiro de 1986,11 pacientes foram submetidos a transplante cardiaco ortotópico. Eram todos do sexo masculino, com idade variando de 39 a 54 anos; 6 com cardiopatia isquêmica, 4 com cardiomiopatia dilatada e um com cardiomiopatia chagásica. Foi realizado estudo hemodinâmico através de catéter de Swan-Ganz, no pré-operatório, no pós-operatório, após estabilizaçăo na unidade de recuperaçáo, e trinta ou mais dias após o transplante. Os dados mostram melhora progressiva em relaçāo ao indice cardiaco, pressão em artéria pulmonar, pressāo de capilar pulmonar, resistência vascular pulmonar e resistência vascular sistêmica. Três dos 11 pacientes apresentaram disfunçâo renal transitória no pós-operatório imediato e que regrediram até o $15^{\circ}$ dia, enquanto que 2 pacientes apresentaram aumento moderado da creatinina plasmática. Apenas 3 pacientes nảo apresentaram qualquer episódio de rejeiçăo; nos demais, esses episódios foram um diagnóstico histológico sem repercussōes clínicas. Complicaçōes infecciosas ocorreram em 9 pacientes e foram de fácil controle clínico. No pós-operatório tardio, a hipertensâo esteve presente em 8 pacientes, sendo mais acentuada em 2 deles. Não houve óbitos, nesta série de pacientes; todos estão assintomáticos e os 6 primeiros estâo trabalhando.

DESCRITORES: transplante cardiaco, humano.

\section{INTRODUÇÃO}

O transplante cardíaco, realizado com sucesso pelo Dr. BARNARD ', em 1967, inaugurou a era dos transplantes cardiacos humanos. O interesse despertado por esse tipo de terapêutica foi muito grande e admite-se que 58 Serviços realizaram 167 transplantes, entre 1968 e 1970 . A alta incidência de rejeição, infecção, ou ambas as complicaçōes, levando à morte dos receptores, fez com que a maior parte dos Centros descontinuasse seus pro- gramas. Dentre os poucos Centros que mantiveram os seus programas, está o Centro Médico da Universidade de Stanford, que, graças à experiência com diagnóstico da rejeição e tratamento dos pacientes, conseguiu melhorar progressivamente os resultados ${ }^{2}$. O uso da ciclosporina, já previamente testado em outros tipos de transplante, desde 1978 , veio trazer benefícios, em termos de diminuiçāo da morbidade e mortalidade do transplante cardía$\mathrm{co}$, quando introduzido por esse mesmo grupo ${ }^{3}$, em 1980.

\footnotetext{
Trabalho realizado no Instituto do Coraçăo do Hospıtal das Clinicas da Faculdade de Medicina da Unıversidade de Sáo Paulo Săo Paulo. SP. Brasil 
STOLF. N. A. G.; BOCCHI, E; LEMOS, P. C. P.; JATENE, F. B.; POMERANTZEFF, P. M. A.; HIGUSHI, L.; KALIL, J.; FIORELLI, A. I. AULER JÜNIOR, J. O. C; BELLOTTI, G.; SILVA, L. A.; PILEGGI, F; JATENE, A. D. - Transplante cardiaco humano: experiência inicial. Rev. Bras. Circ. Cardiovasc. 1(2):34-40, 1986.

O objetivo deste trabalho é a apresentação da experiência inicial de 11 transplantes cardiacos realizados no Instituto do Coração da Faculdade de Medicina da Universidade de São Paulo.

\section{CASUISTICA E RESULTADOS}

No Instituto do Coração, de 12 de março de 1985 a 3 de fevereiro de 1986, 11 pacientes foram submetidos a transplante cardiaco ortotópico. Os dados pré-operatórios sâo apresentados na Figura 1. Verifica-se que as idades variaram de 39 a 54 anos. Todos eram do sexo masculino, sendo os diagnósticos mais freqüentes a cardiomiopatia isquêmica e dilatada. Todos os pacientes estavam em classe funcional IV, sendo que 4 deles tiveram, durante a internaçāo que precedeu o implante, período na unidade coronária, hipotensos e em uso de dobutamina e nitroprussiato de sódio, além de outras drogas. O critério de seleção foi aquele adotado por outros grupos ${ }^{5}$.

os doadores foram pacientes com diagnóstico de morte cerebral e o coração foi retirado em prédio vizinho ao Instituto do Coraçāo, em 1 caso, transportado à distância de cidade de interior do estado, em outro, e retirado no Instituto do Coraçāo, nos demais.

A técnica do transplante cardíaco foi aquela est abslecida por LOWER et alii ${ }^{6}$. O coração do doador foi protegido com injeção de 700 $\mathrm{ml}$ de solução cardioplégica tipo Saint Thomas; em seguid 3, imerso em solução fisiológica a $4^{\circ} \mathrm{C}$. A soluçāo cardioplégica foi repetida na totalidade dos casos.

A imunossupressão foi realizada com ciclosporina e corticóide em 10 pacientes e com ciclosporina e azatioprina no paciente diabético.

Em todos os pacientes, o coração transplantado se recuperou com excelente desempenho. Foi mantido com soluçāo de dopamina, rotineiramente, e nitroprussiato, na maior parte dos, casos. O paciente teve colocado um catéter de Śwan-Ganz para avaliação hemodinâmica. Esse estudo foi realizado no pré-operatório, pós-operatório imediato, após a estabilização do paciente na Unidade de Transplante e diariamente, até a sua retirada do 2. ao 4 dia. $\mathrm{Na}$ época de realizaçāo da biópsia endomiocárdica, foi passado, novamente, o catéter de Swan-Ganz e foram realizadas as mesmas medidas. As Figuras 3 a 7 mostram alguns dos dados hemodinâmicos no pré-operatório, logo após o transplante, no 1: dia de pós-operatório e no período mais tardio, que variou de 30 dias a um ano. Săo apresentados os dados médios e desvio padrăo.

Verifica-se que o índice cardíaco apresenta aumento logo após o transplante e, em geral, um incremento adicional no 1 : dia e tardiamente. A pressão na artéria puimonar, nos seus vários valores, apresenta queda progressiva com a evolução do pós-operatório. Do mesmo modo, cai a pressão em capilar pulmonar. A resistência vascular pulmonar cai, especialmente nos pacientes que já a apresentavam mais elevada; apenas 1 paciente teve importante aumento desse índice, no pós-operatório imediato. Recebeu prostacilina e, posterior-

\begin{tabular}{|c|c|c|c|c|c|c|c|}
\hline CASON: & INICIAIS & $I D A D E$ & SEXO & $C O A$ & $\begin{array}{l}\text { DIAG. } \\
M I O C .\end{array}$ & $\begin{array}{c}\text { CLASSE } \\
\text { FUNCIONAL }\end{array}$ & $\begin{array}{c}D A T A \\
\text { OPEAAÇĀO }\end{array}$ \\
\hline 1 & CF & 50 & $M$ & BR & ISQUEMMICA & IV & 12.03 .85 \\
\hline 2 & MK & 45 & $M$ & AM & DILATADA & IV $\cdots$ & 26.06 .85 \\
\hline 3 & $F A B$ & 48 & $M$ & BR & DILATADA & IV $\cdot$ & 11.07 .85 \\
\hline 4 & BST & 41 & $M$ & AM & ISQUÊMICA & IV & 04.08 .85 \\
\hline 5 & $E A$ & 51 & $M$ & PD & ISQUEAMICA & IV & 04.09 .85 \\
\hline 6 & OMV & 43 & M & PD & CHAGÁSICA & IV $\cdot$ & 16.09 .85 \\
\hline 7 & NG & 54 & M & BR & DILATADA & IV & 03.11 .85 \\
\hline 8 & AAS & 39 & $M$ & BR & ISQUÊMICA & IV & 09.12 .85 \\
\hline 9 & AASN & 46 & M & $\mathrm{BA}$ & ISQUÊMICA & IV & 11.12 .85 \\
\hline 10 & JEH & 44 & $M$ & BA & DILATADA & IV $\cdots$ & 13.01 .86 \\
\hline 11 & APM & 48 & $M$ & BA & ISQUÊMICA & IV & 03.02 .86 \\
\hline
\end{tabular}


STOLF, N. A. G.; BOCCHI, E.; LEMOS, P. C. P.; JATENE, F. B.; POMERANTZEFF, P. M. A.; HIGUSHI, L.; KALIL, J.; FIORELLI, A. I.; AULER JÚNIOR, J. O. C.; BELLOTTI, G.; SILVA, L. A.; PILEGGI, F.; JATENE, A. D. - Transplante cardiaco humano: experiência inicial. Rev. Bras. Circ. Cardiovasc., 1(2):34-40, 1986.

mente, evoluiu com valores normais. A resistência vascular sistêmica também caiu, logo após o transplante, e manteve valores estáveis até o pós-operatório tardio.

Quanto às alteraçōes renais, 1 paciente mostrava aumento significativo da creatinina plasmática no pré-operatório, 3 apresentavam aumento significativo após a operaçāo, sendo que essas alterações se normalizaram até o $15^{\circ}$ dia de pós-operatório, sem que se interrompesse o uso da ciclosporina. Tardiamente, 2 pacientes apresentam aumento moderado e consistente da creatinina plasmática (Figura 7).

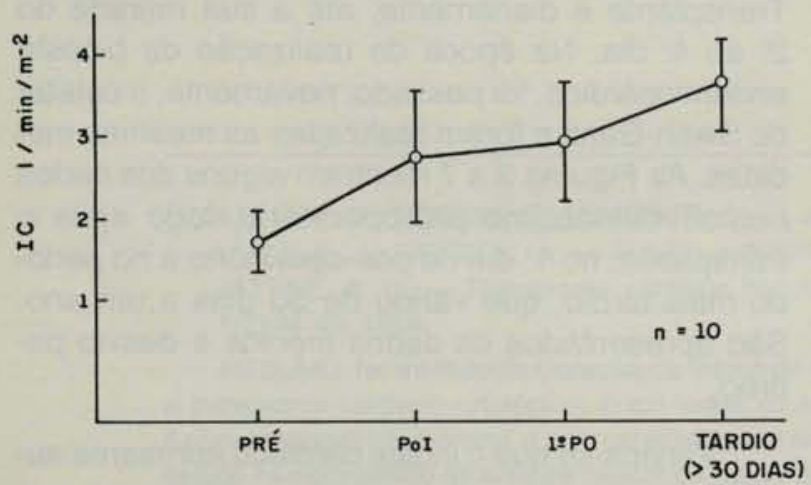

Fig. 2 - Dados hemodinâmicos. Indice cardiaco, média desvio padrão em pré-operatório e pós-operatório.

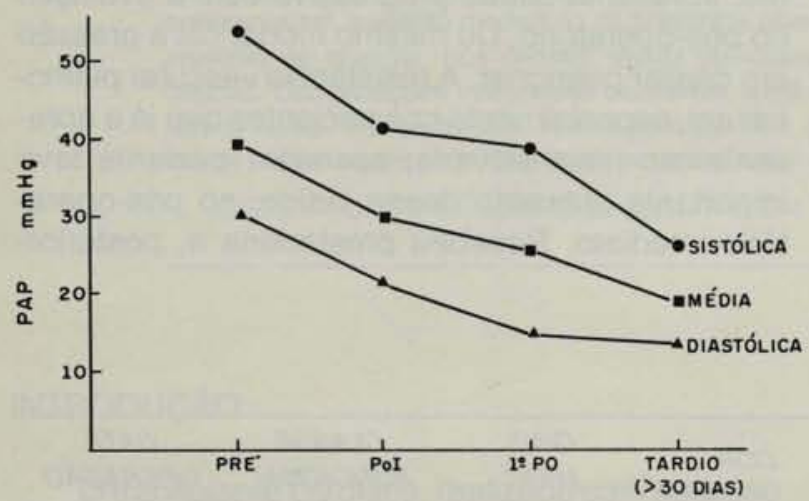

Fig. 3 - Dados hemodinâmicos. Pressão pulmonar, sistólica, diastólica e média no pré-operatório e em 3 periodos do pós-operatório.

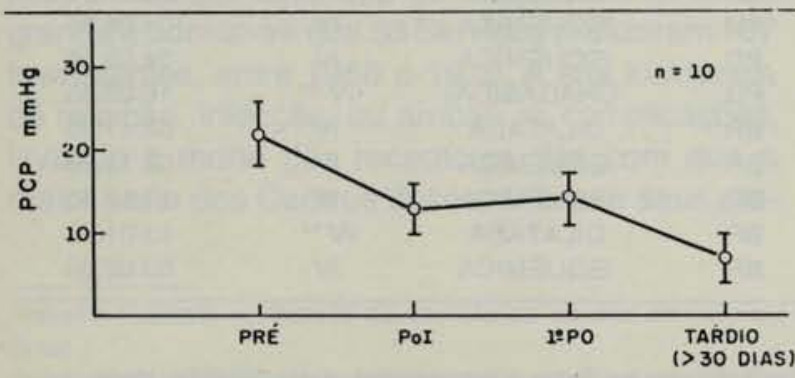

Fig. 4-Dados hemodinâmicos. Pressâo de capilar pulmonar média e desvio padrāo no pré-operatório e pós-operatório.

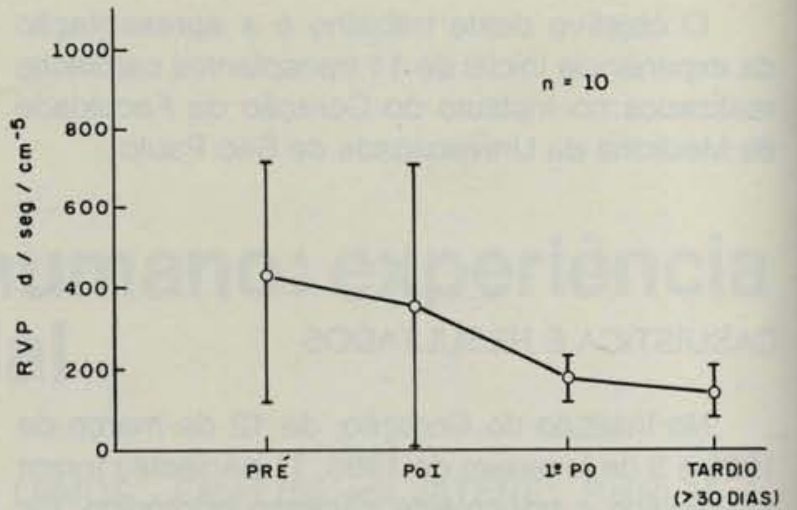

Fig. 5 - Dados hemodinâmicos. Resistência vascular pulmonar média e desvio padrăa no pré-operatório e pós-operatório.

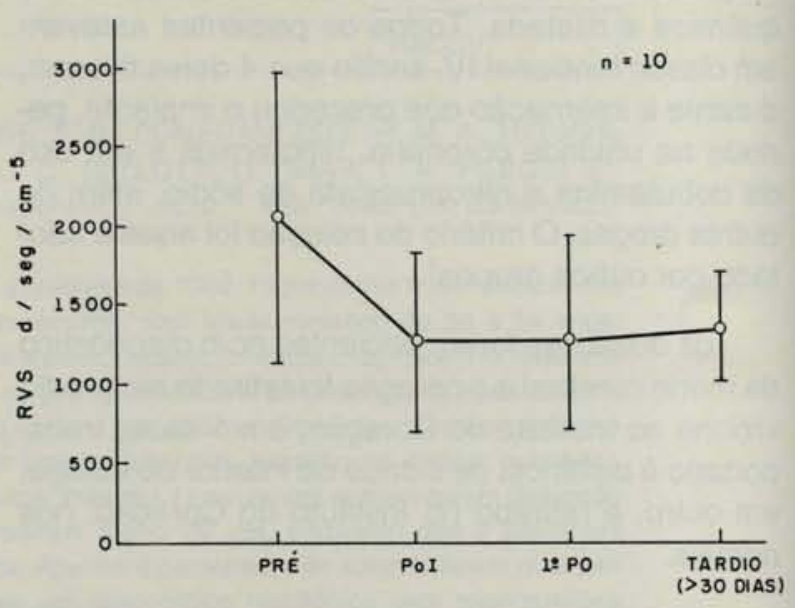

Fig. 6 - Dados hemodinâmicos. Resistência vascular sistêmica média e desvio padrâo no pré-operatório e pós-operatório.

Os pacientes foram submetidos, sistematicamente, a biópsia endomiocardica, semanalmente no 1 ? mês, quinzenalmente até o 3 : mês e, a seguir, mensalmente até o fim do 1 : ano. Segundo os critérios de BILLINGHAM ${ }^{3}$, o diagnóstico histológico da rejeição foi realizado com base no infiltrado linfocitário e presença de necrose de fibras. Os pacientes, quando apresentaram a forma moderada, foram tratados com pulsoterapia de metilprednisoIona $1 \mathrm{~g}$ por via venosa, durante 3 dias. Um paciente recebeu, também, globulina antitimocitária em um período de rejeição persistente. A Figura 8 mostra apenas 3 pacientes que não apresentaram qualquer episódio de rejeição; os demais apresentaram um, ou mais, episódios em períodos variáveis do pós-operatório. Vale ressaltar que esses episódios foram de diagnóstico histológico; os pacientes não apresentaram qualquer repercussão clínica, nem mesmo na avaliação hemodinâmica já descrita. 
STOLF, N. A. G.; BOCCHI; E.; LEMOS, P. C. P.; JATENE, F. B.; POMERANTZEFF, P. M. A.; HIGUSHI, L.; KALIL, J.; FIORELLI, A. I.; AULER JÚNIOR, J. O. C.; BELLOTTI, G.; SILVA, L. A.; PILEGGI, F.; JATENE, A. D. - Transplante cardiaco humano: experiência inicial. Rev. Bras. Circ. Cardiovasc., 1(2):34-40, 1986

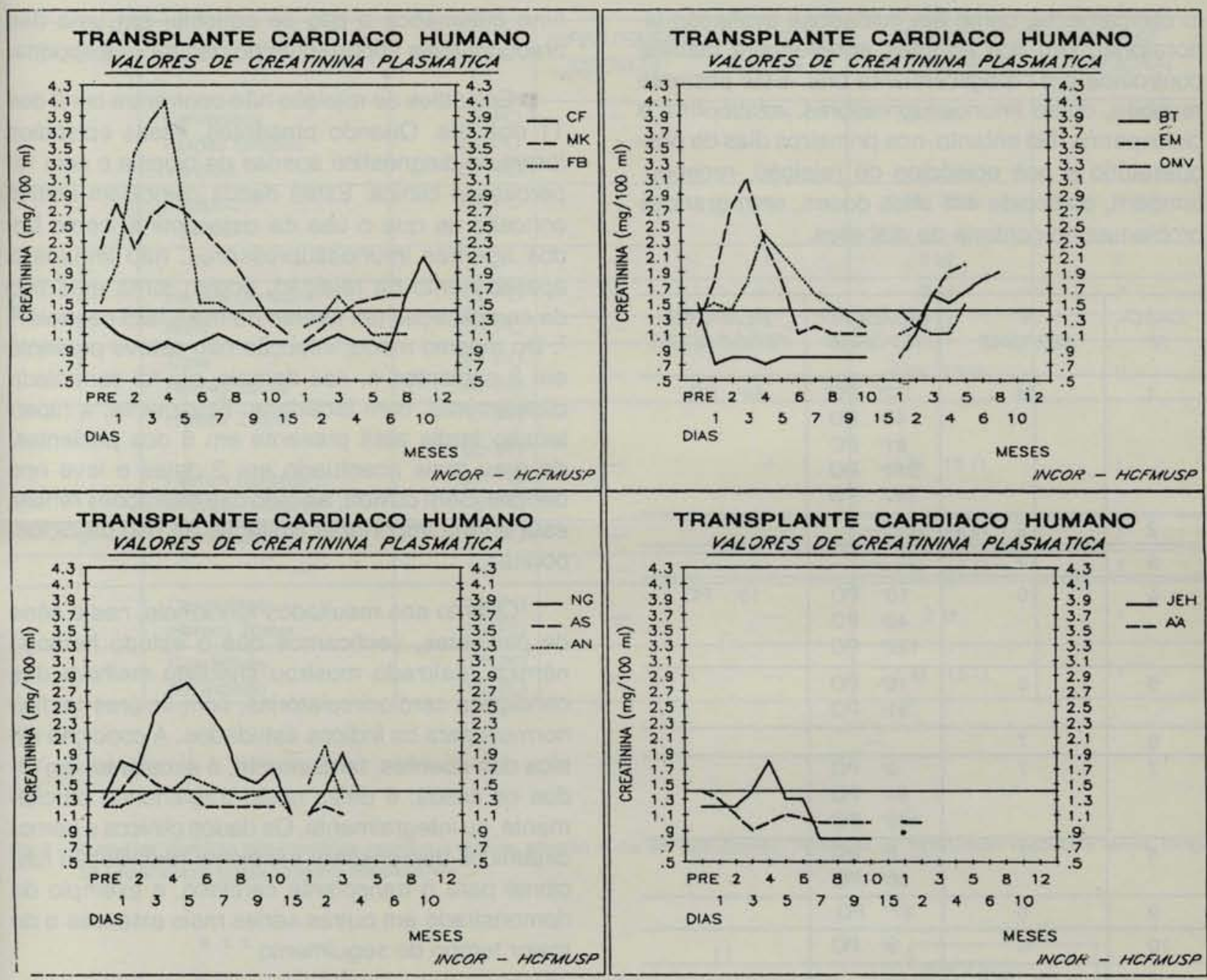

Fig. 7 - Valores de creatinina plasmática dos 11 pacientes nos primeiros 15 dias de pós-operatório e um mês ou mais após.

A Figura 9 mostra a incidência de duas outras importantes complicações. Quanto à infecção, verificamos que 2 pacientes não apresentaram nenhum episódio e os demais apresentaram um ou mais episódios. A hipertensão, no pós-operatório tardio, como efeito colateral da ciclosporina, ocorreu em todos, com excessão de 3 pacientes. Em 2 deles, ela foi mais acentuada, sendo controlada com um medicamento, ou associação de medicamentos.

O seguimento variou de 1 mês e 15 dias a 12 meses e os pacientes estão assintomáticos, realizando exercício físico orientado e os 6 primeiros pacientes estão trabalhando parcialmente, ou integralmente.

\section{DISCUSSÃO}

Após os resultados desapontadores iniciais, a sobrevida vem aumentando progressivamente $e$ a morbidade vem diminuindo, no transplante cardíaco humano.

Vários avanços têm sido relacionados com essa melhora. Alem da experiência acumulada com o manejo desse tipo de paciente, deve-se destacar: 1) o refinamento na indicação; 2) melhora na proteção miocárdica e transporte do coração à distância; 3) novos agentes imunossupressores, especialmente a ciclosporina; 4) a introdução, em 1972, da técnica da biópsia endomiocárdica.

Quanto à indicação, na série apresentada, verifica-se predominância de casos com cardiomiopatia isquêmica e dilatada e não há casos de outras cardiopatias, como a valvar, que também é indicação mais rara em todas as casuísticas ${ }^{2}$, 5. 9 . De particularidade, nota-se apenas o transplante em 1 paciente com cardiomiopatia chagásica (caso $n$ : 6), que, com mais de seis meses de evolução, não apresenta evidências de reativação da doença, tan- 
STOLF, N. A. G.; BOCCHI, E.; LEMOS, P. C. P.; JATENE, F. B.; POMERANTZEFF, P. M. A.; HIGUSHI, L.; KALIL, J.; FIORELLI, A. I.; AULER JÜNIOR, J. O. C.; BELLOTTI, G.; SILVA, L. A.; PILEGGI, F.; JATENE, A. D. - Transplante cardiaco humano: experiência inicial. Rev. Bras. Circ. Cardiovasc., 1(2):34-40, 1986.

to clinicamente, como em cuidadosa avaliação laboratorial. Um dos doentes apresentava diabete controlada por hipoglicemiante oral. Este paciente recebeu, como imunossupressores, azatioprina e ciclosporina. No entanto, nos primeiros dias de pósoperatório e nos episódios de rejeição, recebeu, também, corticóide em altas doses, sem grandes problemas no controle de diabetes.

\begin{tabular}{|c|c|c|c|}
\hline $\begin{array}{c}\text { CASO } \\
N^{\circ}\end{array}$ & $\begin{array}{c}N^{\circ} \\
\text { BIOPSIAS }\end{array}$ & $\begin{array}{l}\text { REJEICAAO } \\
P O-G R A U\end{array}$ & $\begin{array}{c}\text { REJEIÇÃO } \\
\text { PERSISTENTE }\end{array}$ \\
\hline 1 & 14 & $\begin{array}{rr}37^{\circ} & \mathrm{PO} \\
44^{\circ} & \mathrm{PO} \\
81^{\circ} & \mathrm{PO} \\
240^{\circ} & \mathrm{PO} \\
280^{\circ} & \mathrm{PO}\end{array}$ & $44^{\circ} \mathrm{PO}{ }^{\circ}$ \\
\hline 2 & 9 & - & \\
\hline 3 & 12 & - & \\
\hline 4 & 10 & $\begin{array}{rr}10^{\circ} & \mathrm{PO} \\
40^{\circ} & \mathrm{PO} \\
122^{\circ} & \mathrm{PO}\end{array}$ & $18^{\circ} \mathrm{PO}$ \\
\hline 5 & 9 & $\begin{array}{ll}16^{\circ} & \mathrm{PO} \\
91^{\circ} & \mathrm{PO} \\
\end{array}$ & \\
\hline 6 & 7 & - & \\
\hline 7 & 7 & $\begin{array}{rr}9^{\circ} & \mathrm{PO} \\
81^{\circ} & \mathrm{PO} \\
108^{\circ} & \mathrm{PO} \\
\end{array}$ & \\
\hline 8 & 7 & $\begin{array}{rr}8^{\circ} & \mathrm{PO} \\
50^{\circ} & \mathrm{PO} \\
\end{array}$ & . \\
\hline 9 & 6 & $37^{\circ} \mathrm{PO}$ & \\
\hline 10 & 5 & $9^{\circ} \quad \mathrm{PO}$ & 11 \\
\hline 5 & $\begin{array}{ll}10^{\circ} & \mathrm{PO} \\
24^{\circ} & \mathrm{PO}\end{array}$ & $31^{\circ} \mathrm{PO}$ & \\
\hline
\end{tabular}

- Puiso + ATG

* Não tratada

Fig. 8-Dados relativos a rejeiçăo mostrando o n: de biópsias e o dia de pós-operatório em que ocorreu o episódio de rejeição e se ele persistiu

Vários problemas e complicações, ligados ao transplante propriamente dito, ou à imunossupressão, são já bem conhecidos. Alterações renais no pós-operatório imediato ocorreram em 6 pacientes e se resolveram, ao fim de 15 dias, apenas com a diminuição da dose de ciclosporina, como referido por outros ${ }^{3 .}{ }^{7}$. Não houve caso de complicação renal mais grave e, tardiamente, 3 pacientes apresentavam níveis discretamente elevados de creati- nina plasmática e isto se constitui em uma das preocupaçōes importantes do uso da ciclosporina.

Episódios de rejeição não ocorreram em 3 dos 11 doentes. Quando presentes, esses episódios foram de diagnóstico apenas da biópsia e sem repercussão clínica. Estes dados coincidem com o conceito de que o uso da ciclosporina, como um dos agentes imunossupressores, não impede o aparecimento da rejeição, porém torna este tipo de complicação um evento de mais fácil controle ${ }^{3 .}$ 7. Do mesmo modo, infecção não esteve presente em 2 pacientes e, nos demais, ela foi controlada clinicamente, com facilidade. Finalmente, a hipertensão tardia está presente em 8 dos pacientes, de grau mais acentuado em 2 deles e leve nos demais. Sem dúvida, ao lado das alterações renais, esta é uma importante limitação ao uso da ciclosporina $^{7}$.

Quanto aos resultados funcionais, neste série de pacientes, verificamos que o estudo hemodinâmico realizado mostrou imediata melhora das condiçōes cardiocirculatórias, com valores tardios normais para os índices estudados. A condição clínica dos doentes, tardiamente, é excelente, em todos os casos: 6 deles estão trabalhando parcialmente, ou integralmente. Os dados clínicos e hemodinâmicos demonstram excelente reabilitação funcional para o transplante cardíaco, a exemplo do demonstrado em outras séries mais extensas e de maior tempo de seguimento ${ }^{2,7,9}$.

Considerando que os dados da literatura mostram que os pacientes indicados para transplante, em que este, por várias razões, não foi realizado, morrem, na sua imensa maioria, até os três meses, poucos sobrevivem mais de seis meses e nenhum mais de um ano. Os resultados obtidos na série de 11 pacientes no Instituto do Coração mostram que este tipo de procedimento prolongou a vida e melhorou a qualidade de vida dos pacientes.

Em relação à sobrevida, verifica-se que as séries mais recentes, especialmente com protocolos que incluem a ciclosporina, apresentam sobrevida em torno de $80 \%$ ao fim de um ano, $70 \%$, ao fim de três anos e provável sobrevida em torno de $60 \%$ ao fim de cinco anos ${ }^{7.8 .9}$. A série apresenta 9 pacientes com mais de três meses de evolução, que é o período de maior risco, sem nenhum óbito, e faz antecipar um bom resultado, em termos de sobrevida. 
STOLF, N. A. G.; BOCCHI, E.; LEMOS, P. C. P.; JATENE, F. B.; POMERANTZEFF, P. M. A.; HIGUSHI, L.; KALIL, J.; FIORELLI, A. I.; AULER JÜNIOR, J. O. C.; BELLOTTI, G.; SILVA, L. A.; PILEGGI, F.; JATENE, A. D. - Transplante cardiaco humano: experiência inicial. Rev. Bras. Circ. Cardiovasc., 1(2):34-40, 1986.

\begin{tabular}{|c|c|c|c|c|c|c|}
\hline CASON: & INFECÇĀO & TIPO - PO & $\begin{array}{l}\text { HIPERTENSĀO } \\
\text { IMEDIATA }\end{array}$ & $-\underset{T A R D I A}{-G R A U}$ & $\begin{array}{c}\text { TEMPO } \\
\text { SEGUIMENTO }\end{array}$ & $\begin{array}{c}\text { CLASSE } \\
\text { FUNCIONAL }\end{array}$ \\
\hline 1 & $\begin{array}{l}\text { Candidiase } \\
\text { Herpes Simples } \\
\text { Varicela }\end{array}$ & $\begin{array}{r}5^{\circ} \mathrm{PO} \\
29^{\circ} \mathrm{PO} \\
60^{\circ} \mathrm{PO}\end{array}$ & + & +++ & $12 \mathrm{M}$ & $1^{\circ}$ \\
\hline 2 & $\begin{array}{l}\text { Abcesso } \\
\text { Perineal }\end{array}$ & $15^{\circ} \mathrm{PO}$ & - & + & $8 \mathrm{M} 25 \mathrm{D}$ & $1^{*}$ \\
\hline 3 & - & - & 一 & + & $8 \mathrm{M}$ & $1^{*}$ \\
\hline 4 & Herpes Genital & $15^{\circ} \mathrm{PO}$ & - & ++ & $7 \mathrm{M}$ & $1^{*}$ \\
\hline 5 & Acne & $33^{\circ} \mathrm{PO}$ & - & +++ & $6 \mathrm{M}$ & $\mathrm{I}^{*}$ \\
\hline 6 & $\begin{array}{l}\text { Acne } \\
\text { Herpes Zoster }\end{array}$ & $\begin{array}{r}20^{\circ} \mathrm{PO} \\
180^{\circ} \mathrm{PO} \\
\end{array}$ & - & + & $6 \mathrm{M} 15 \mathrm{D}$ & $1^{*}$ \\
\hline 7 & $\begin{array}{l}\text { Herpes Zoster } \\
\text { Herpes Simples } \\
\text { Infecção Urinária }\end{array}$ & $\begin{array}{r}7^{\circ} \mathrm{PO} \\
90^{\circ} \mathrm{PO} \\
27^{\circ} \mathrm{PO} \\
5^{\circ} \mathrm{PO} \\
\end{array}$ & - & + & $4 \mathrm{M} \quad 15 \mathrm{D}$ & 1 \\
\hline 8 & Candidiase & $23^{\circ} \mathrm{PO}$ & - & 一 & $3 \mathrm{M} 15 \mathrm{D}$ & 1 \\
\hline 9 & - & - & - & + & $2 \mathrm{M} 15 \mathrm{D}$ & 1 \\
\hline 10 & $\begin{array}{l}\text { Candidiase } \\
\text { Herpes Zoster }\end{array}$ & $\begin{array}{l}12^{\circ} \mathrm{PO} \\
20^{\circ} \mathrm{PO}\end{array}$ & - & - & $2 \mathrm{M}$ & 1 \\
\hline 11 & $\begin{array}{l}\text { Candidíase } \\
\text { Inf. Incisão }\end{array}$ & $\begin{array}{r}7^{\circ} \mathrm{PO} \\
15^{\circ} \mathrm{PO} \\
\end{array}$ & - & - & $1 \mathrm{M} 15 \mathrm{D}$ & I \\
\hline
\end{tabular}

\section{- Trabalhando}

Fig. 9 - Dados da evoluçăo pós-operatória relativos a tipo de infecção e dia em que ocorreu, incidência de hipertensāo imediata e tardia, tempo de seguimento e classe funcional dos pacientes.

RBCCV

STOLF, N. A. G.; BOCCHI, E.; LEMOS, P. C. P.; JATENE, F. B.; POMERANTZEFF, P. M. A.; HIGUSHI, L.; KALIL, J.; FIORELLI, A. I.; AULER Jr., J. O. C.; BELLOTTI, G.; SILVA, L. A.; PILEGGI, F.; JATENE, A. D. - Human cardiac transplant: initial experience. Rev. Bras. Cir. Cardiovasc., 1 (2): 34-40, 1986.

ABSTRACT: At the Instituto do Coração, University of São Paulo Medical School, 11 patients were submitted to heart transplantation from march 1985 up to february 1986. All were male, with ages of 39-59 years, 6 with coronary heart disease, 4 with dilated cardiomyopathy and 1 with Chagas cardiomyopathy. The patients were studied hemodynamically with a Swan-Ganz catheter pre-operatively, at the arrival in the intensive care unit, in the first postoperative day and 30 or more days after the transplant. The data showed that there was a progressive increase of cardiac index and decreases of pulmonary artery pressure, capilary pulmonary wedge pressure, pulmonary vascular resistance and systemic vascular resistance. Three of the 11 patients had immediate renal dysfunction that returned to normal by the $15^{\text {th }}$ day. Late postoperatively 2 patients had increase of creatinine levels. Only 3 patients had no rejection episodes; among the others these episodes were represented by hystological alterations with no clinical manifestations. Infections complications occurred in 9 patients and were easily clinically treated. Late postoperatively, hypertension was present in 8 patients; in 2 of them it was moderate. There was no death in these 11 patients; all are symptom free and the first 6 are working.

DESCRIPTORS: heart transplantation, human.

\section{REFERÊNCIAS BIBLIOGRÁFICAS}

1 BARNARD, C. N. - A human cardiac transplant: an interim report of a successful operation performed at Groote Schuur-Hospital, Cape Towun. S. Afr. Med. J., 41 (48): 1271-1274, 1967.
2 BAumgaRtNeR, W. A.; ReITZ, B. A.; BIEBER, C. P.; OYER, P. E.; SHUMWAY, N. E.; STINSON, E. B. Current expectation in cardiac transplantation. J. Thorac. Cardiovasc. Surg., 75 (4): 525-530, 1978. 
STOLF, N. A. G.; BOCCHI, E.; LEMOS, P. C. P.; JATENE, F. B.; POMERANTZEFF, P. M. A.; HIGUSHI, L.; KALIL, J.; FIORELLI, A. I.; AULER JÜNIOR, J. O. C.; BELLOTTI, G.; SILVA, L. A.; PILEGGI, F.; JATENE, A. D. - Transplante cardiaco humano: experiência inicial. Rev. Bras. Circ. Cardiovasc., 1(2):34-40, 1986.

3 BILLINGHAM, M. E. - Diagnosis of cardiac rejection by endomyocardial biopsy. Heart Transplant, 1 (1): 25-30, 1981.

4 JAMIESON, S. W. - Recent developments in heart and heart-lung transplantation. Transplant. Proc., 17 (1): 199-203, 1985.

5 JAMIESON, S. W.; OYER, P. E.; REITZ, B. A.; BAUMGARTNER, W. A.; BIEBER, C. P.; STINSON, E. B.; SHUMWAY, N. E. - Cardiac transplantation at Stanford. Heart Transplant., 1(1):86-91, 1982.

6 LOWER, R. R.; DONG, E.; SHUMWAY, N. E. - Long-term survival of cardiac homografts. Surgery, 58 (1): $110-119,1965$.
7 OYER, P. E.; STINSON, E. B.; JAMIESON, S. W.; HUNT, S.; REITZ, B. A.; BIEBER, C. P.; SCHOEDER, J.; BILLINGHAM, M.; SHUMWAY, N. E. - One year experience with cyclosporine $A$ in clinical heart transplantation. Heart Transplant, 1 (4):285-290, 1982

8 STINSON, E. B. - Overal review of the Stanford program and results. In: Cardiac e transplantation at Stanford. Stanford University, Stanford, USA, 7-9 out. 1985.

9 YACOUB, M.; ALIVIZATOS, P.; KHAGHANI, A.; MITCHELL, A. - The use of cyclosporine, azathioprine, and antithymocyte globulin with or without low-dose steroids for immunosuppression of cardiac transplant patients. Transplant. Proc., 17 (1): 221-222, 1985. 